\title{
Das Myalgie-Faszikulations-Krampus-Syndrom (MFKS)
}

\section{The cramp-fasciculation-syndrome (CFS)}

\author{
Autoren \\ Jens D. Rollnik ${ }^{1}$, Jan Däuper ${ }^{1}$ \\ Institute \\ 1 Institut für neurorehabilitative Forschung (InFo), Medizini- \\ sche Hochschule Hannover, BDH-Klinik Hess. Oldendorf \\ gGmbH
}

Schlüsselwörter

Myalgie-Faszikulations-Krampus-Syndrom, Pathophysiologie, Differenzialdiagnostik, Therapie

Key words

cramp-fasciculation-syndrome, pathophysiology, differential diagnosis, therapy

eingereicht $\quad 05.07 .2019$

akzeptiert 25.09.2019

Bibliografie

DOI https://doi.org/10.1055/a-1019-7646

Fortschr Neurol Psychiatr 2020; 88: 459-463

(c) Georg Thieme Verlag KG Stuttgart · New York

ISSN 0720-4299

Korrespondenzadresse

Prof. Dr. med. Jens D. Rollnik

Institut für neurorehabilitative Forschung (InFo), Medizinische

Hochschule Hannover

BDH-Klinik Hess. Oldendorf gGmbH

Greitstr. 18-28

31840 Hessisch Oldendorf

Tel.: 05152781231

Fax: 05152781198

E-Mail: prof.rollnik@bdh-klinik-hessisch-oldendorf.de

\section{ZUSAMMENFASSUNG}

Die vorliegende Übersichtsarbeit beschäftigt sich mit dem Myalgie-Faszikulations-Krampus-Syndrom (MFKS), einer gutartigen Erkrankung, die zu den Hyperexzitabilitätssyndromen des peripheren Nervensystems gezählt wird. Der Artikel stellt die Klinik, Pathophysiologie, Differenzialdiagnostik und Therapie dar und illustriert das MFKS mit einem typischen Fallbericht.

\section{ABSTRACT}

The present review focuses on the cramp-fasciculation syndrome, a benign disorder which is regarded as a hyperexcitability syndrome of the peripheral nervous system. The article presents clinical features, pathophysiology, differential diagnosis, therapy and a case report to illustrate the cramp-fasciculation-syndrome.

\section{Einleitung}

Das Myalgie-Faszikulations-Krampus-Syndrom (MFKS) („crampfasciculation-syndrome“) erstmals 1948 von Denny-Brown und Foley beschrieben [1], wird auch als benigne Motoneuron-Erkrankung bezeichnet. Die klinische Symptomatik ist durch den deutschen Begriff schon hinreichend bezeichnet: Im Vordergrund der Symptomatik stehen Myalgien und Krampi, begleitet von Faszikulieren an den Extremitäten. Unter einem Krampus versteht man sichtbare, palpable und schmerzhafte Kontraktionen eines Muskels oder von Teilen eines Muskels, spontan auftretend oder nach
Willkürinnervation eingeleitet [2]. Das Auftreten korreliert dabei nicht mit der Stärke der auslösenden Kontraktion und kann durch passive Dehnung des Muskels beendet werden [2]. Die Myalgien sind belastungsinduziert und betreffen vor allem die Extremitätenmuskulatur [2], bevorzugt Oberschenkel und Waden.

Faszikulationen sind spontane, unwillkürliche Entladungen einer Gruppe von Muskelfasern, die entweder eine ganze motorische Einheit oder Teile repräsentieren [3]. Benigne Faszikulationspotenziale (FP) lassen sich in der Elektromyografie (EMG) nicht von solchen pathologischen Ursprungs differenzieren [4]. 
Faszikulationen kommen bei Gesunden häufig vor, z. B. im M. orbicularis oculi. In einer Studie konnten FP bei gesunden Erwachsenen mittels Sonografie bei $43 \%$ im M. abductor hallucis festgestellt werden [5]. In einer anderen Untersuchung an Gesunden mittels Oberflächen-EMG-Ableitung ließen sich sogar in $77 \% \mathrm{FP}$ nachweisen [6]. Klinisch werden benigne Faszikulationen nicht von einer Muskelatrophie oder anderen neurologischen Auffälligkeiten begleitet. Wegen der Differenzialdiagnose einer Motoneuron-Erkrankung lösen Faszikulationen vor allem unter Ärzten Ängste aus, wenngleich sich in einer prospektiven Untersuchung nur in $5 \%$ der Fälle tatsächlich eine Amyotrophe Lateralsklerose (ALS) entwickelte [7]. Klinisch können gutartige Faszikulationen sporadisch zumeist an den Unterarmen, Waden und am Daumen beobachtet werden [8]. Sie können durch Koffein, Nikotin, Stress, Angst und anstrengende körperliche Tätigkeiten evoziert werden [8]. Benigne Faszikulationen zeigen sich oft über viele Jahre, ohne dass es zu neurologischen Ausfällen kommt [8].

Die Liste von Ursachen pathologischer Faszikulationen ist in -Tab. 1 wiedergegeben.

-Tab. 1 Ursachen von pathologischen Faszikulationen [4, 8, 9].

- Vorderhornerkrankungen: Amyotrophe Lateralsklerose, spinale Muskelatrophien

Polyneuropathien (insbesondere entzündliche Formen), Multifokale Motorische Neuropathie (MMN), Radikulopathien, Plexusläsionen, Mononeuritiden, Syringomyelie

- Hyperexzitabilitätssyndrome: Neuromyotonie (Isaacs-Syndrom, Morvan-Syndrom)

- Hereditäre Erkrankungen: hereditäre Neuropathien, Spinozerebelläre Ataxien, GM2-Gangliosidose (lysosomale Speicherkrankheit), Morbus Fabry, Gerstmann-Sträussler-Krankheit, Rett-Syndrom, HuntingtonErkrankung, familiäre Amyloidose

- Paraneoplastische Erkrankungen: Anti-Hu-Syndrom

- Infektiöse Erkrankungen: Poliomyelitis, Creutzfeldt-Jakob-Erkrankung, HIV, Syphilis, Tetanus

- Metabolische Erkrankungen: Thyreotoxikose

- Intoxikationen / Medikamente: Vergiftungen mit Organophosphorverbindungen und Phosphorsäureester, Lithium, Kortikosteroide

\section{Pathophysiologie}

Das MFKS wird zu den Hyperexzitabilitätssyndromen des peripheren Nervensystems gezählt, d. h. zu Erkrankungen, die mit Faszikulationen, Myokymien (langsame Kontraktionen in wechselnden Gruppen von Muskelfasern, die kaum einen Bewegungseffekt haben, aber an der Hautoberfläche sichtbar werden), Neuromyotonie, Krampi oder Tetanie einhergehen können [9]. Faszikulationen entstehen durch spontane Entladungen motorischer Einheiten [9], wobei vermutlich spannungsabhängige Kaliumkanäle (voltage gated potassium channel, VGKC) involviert sind [10]. Antikörper gegen VGKC führen zu einer Hyperexzitabilität peripherer Nerven [11]. Antineuronale Antikörper finden sich allerdings nur bei jedem dritten MFKS-Patienten [12]. VGKC-Antikörper sind auch in der Pathophysiologie neuromyotoner Krankheitsbilder involviert, insbesondere dem Isaacs-Syndrom und dem Morvan-Syndrom, bei dem es neben der Neuromyotonie auch zu einer limbischen Enzephalitis kommt [13]. Unter der heterogenen Gruppe von VGKC-Antikörpern kommt in der Pathophysiologie des MFKS als Antigen-Target vor allem das paranodale Kaliumkanal-assoziierte Protein CASPR2 (contactin-associated protein-like 2) infrage [14]. Autoantikörper gegen CASPR2 führen zu einer elektrischen Instabilität peripherer Nerven [14]. Neben den VGKC wurde kürzlich noch ein weiteres mögliches Antigen identifiziert, das TRPA1 (transient receptor potential ankyrin A1), ein Kationenkanal, dessen Funktion noch nicht abschließend geklärt ist [15]. Mutationen im TRPA1-Gen führen zu einem Hypersensitivitäts- bzw. -exzitabilitätssyndrom mit MFKS, Asthma bronchiale, Refluxkrankheit, Migräne, Restless-Legs-Syndrom, Tremor, Hyperalgesie (v.a. auf Kältereize) und kardialen Reizleitungsstörungen [15]. Letztere Symptome finden sich phänotypisch jedoch nur bei einer kleineren Fraktion von MFKS-Patienten.

Wie bereits ausgeführt, liegen antineuronale Antikörper nur in etwa $30 \%$ der MFKS-Patienten vor [12]. Die Pathophysiologie bei Patienten ohne solche Autoantikörper ist bisher nicht geklärt.

Unklar ist auch, ob eine benigne Motoneuron-Erkrankung im Verlauf in seltenen Fällen in eine ALS übergehen kann, oder ob vielmehr die Vorboten einer ALS, insbesondere die generalisierten Faszikulationen, als MFKS fehlinterpretiert wurden. Für eine mögliche Transformation der im Allgemeinen als benigne angesehenen Erkrankung gibt es nur wenige Fallberichte, die keine abschließende Bewertung zulassen [16-18]. In einer älteren Publikation wird über 121 Patienten mit einem benignen Faszikulationssyndrom berichtet, bei denen katamnestisch 2-32 Jahre nach Diagnosestellung kein einziger Patient eine ALS entwickelt hatte [19]. In der zitierten Arbeit findet sich auch ein Hinweis darauf, dass benigne Faszikulationen nach akuten, vornehmlich viralen Infekten auftreten können: Immerhin 19 Patienten (15,7\%) berichteten, dass im Monat vor dem erstmaligen Auftreten von Faszikulationen ein viraler Infekt des oberen Respirationstrakts oder gastrointestinale Beschwerden aufgetreten waren [19]. Es wird in der Veröffentlichung auch der eindrückliche Fall eines 40-jährigen Neurologen beschrieben, der eine akute Gastroenteritis mit Fieber, Erbrechen und Diarrhoe über 48 Stunden Dauer erlitten hatte und drei Tage nach dem Infekt generalisierte Faszikulationen ohne neurologische Ausfälle entwickelte, die auch nach vier Jahren noch persistierten [19].

\section{Diagnostik und Differenzialdiagnostik}

Die Diagnose eines MFKS ist klinisch zu stellen. An sich stellt das MFKS eine Ausschlussdiagnose dar. Differenzialdiagnostisch ist in Anbetracht des Leitsymptoms Faszikulation eine breite Palette von Erkrankungen ( $\triangleright$ Tab. 1) zu berücksichtigen.

Die klinisch-neurophysiologische Diagnostik ist wichtig, v.a. um Erkrankungen auszuschließen, die mit akuter oder chronischer Denervierung einhergehen ( $\bullet$ Tab. 1). Sind ausgeprägte axonale Schädigungszeichen im EMG vorhanden (Fibrillationen und Positive Scharfe Wellen), muss die Diagnose eines MFKS infrage gestellt werden.

Bereits 1991 wurde ein Verfahren der repetitiven Stimulation zur Diagnosestellung eines MFKS publiziert [20]. Die Ableitung erfolgt mit Oberflächenelektroden vom M. abductor hallucis. Zunächst wird der Muskel in Ruhe (baseline) abgeleitet, dann erfolgt eine repetitive Stimulation (fünf supramaximale Reize), zunächst 
mit $0,5 \mathrm{~Hz}$, dann mit 1, 2 und $5 \mathrm{~Hz}$ [20]. In einer Studie wiesen $81 \%$ der MFKS-Patienten eine Hyperexzitabilität in der repetitiven Stimulation auf [21]. Eine höherfrequente repetitive Stimulation mit $10 \mathrm{~Hz}$ ist möglicherweise sensitiver [12].

Die Bestimmung von VGKC-Komplex-Antikörpern ist zu empfehlen, auch wenn nur etwa ein Drittel der Patienten antineuronale Antikörper aufweist [12]. Neben VGKC-Antikörpern kann eine weitergehende immunologische Untersuchung erfolgen, z. B. auf NMDA-Rezeptor-Antikörper (NMDA = N-Methyl-D-Aspartat) und anti-Hu-Antikörper [22]. Allerdings sind Signifikanz und diagnostischer Nutzen noch nicht abschließend bewertbar, da größere Studien fehlen.

\section{Therapie}

Ein Ansprechen, v.a. der als störend empfundenen Faszikulationen, wurde schon früh auf Carbamazepin beschrieben [20]. Insbesondere Patienten mit der bereits oben beschriebenen TRPA1Variante scheinen auf Carbamazepin anzusprechen [15].

In einer Publikation wird eine gute Wirkung des membranstabilisierenden Gabapentins beschrieben, das mit dreimal 300-600 mg/d eingesetzt wurde [23].

Gegen Krampi kann neben hochdosierten Magnesium-Präparaten auch Chininsulfat $200 \mathrm{mg} 1-2$ Tbl. z. N. erwogen werden [2]. Da Chininsulfat neben Herzrhythmusstörungen, Tinnitus, Hör- und Sehstörungen auch potenziell tödliche immunallergische Thrombozytopenien sowie ein hämolytisch-urämisches Syndrom bzw. eine thrombotisch-thrombozytopenische Purpura auslösen kann [24], sollte die Verordnung aber zurückhaltend und nur bei hohem Leidensdruck erfolgen.

Beim Isaacs-Syndrom wurde auch Baclofen off-label eingesetzt, sodass sich eine Wirksamkeit auf die Hyperexzitabilität auch beim MFKS zeigen könnte [25].

\section{Kasuistik}

Ein 50-jähriger Arzt mit generalisierten Faszikulationen stellt sich in der Sprechstunde vor. Erstmalig sei eine störende „Unruhe in der Muskulatur“ vor etwa 25 Jahren aufgetreten. Damals sei eine elektromyografische Untersuchung durchgeführt worden, die neben Faszikulationen keinen Hinweis auf akute oder chronische Denervierung erbracht habe. Er habe befürchtet, an einer ALS erkrankt zu sein. Nach 25-jährigem Verlauf ohne auftretende Paresen sei er aber dahingehend beruhigt. Die Faszikulationen würden ihn stören, v.a. nachts (aber ohne Restless-Legs-Symptomatik), er verspüre eine ständige Unruhe der Muskulatur. Eine Verstärkung der Symptome trete durch schwere körperliche Arbeit, Schlafentzug, Stress, Alkohol-, Kaffee- und Nikotingenuss ein. Nach dem Joggen habe er zudem manchmal muskelkaterartige Beschwerden. Selten würden auch Krampi auftreten, z. B. bei längeren Telefonaten, wenn er den Hörer ans Ohr halte, komme es zu schmerzhaften Verkrampfungen am M. biceps brachii rechts. In der weiteren Vorgeschichte findet sich neben einer Refluxösophagitis noch ein allergisches Asthma bronchiale (in der Jugend). Herzrhythmusstörungen werden verneint. Die Familienanamnese ist leer für neurologische Erkrankungen, allerdings habe die 79-jährige, ansonsten gesunde Mutter bisweilen auch Faszikulationen verspürt.
Therapeutisch wurde bereits Carbamazepin versucht, das zwar geholfen habe, wegen Nebenwirkungen (Schwindel, Übelkeit, Hyponatriämie) aber rasch wieder habe abgesetzt werden müssen.

In der klinischen Untersuchung zeigen sich symmetrisch v.a. an den Waden, aber auch am M. biceps brachii reichlich Faszikulationen, die sich durch Perkussion verstärken lassen. Die weitere Untersuchung erbringt keine Atrophien, keine manifesten oder latenten Paresen, lebhafte Muskeleigenreflexe bei negativen Pyramidenbahnzeichen, keine Beeinträchtigung der epikritischen oder protopathischen Empfindungsqualitäten. Elektroneurografisch ergeben sich keine Hinweise auf eine Polyneuropathie (N. suralis rechts: $63 \mathrm{~m} / \mathrm{s}$, Amplitude der SNAP 5,4 $\mu \mathrm{V}$; N. peroneus rechts: distal motorische Latenz 5,0 ms, Amplitude der MSAP 3,5 mV, NLG $44 \mathrm{~m} / \mathrm{s}$ am Unterschenkel, Amplitude 3,2 $\mathrm{mV}, 47 \mathrm{~m} / \mathrm{s}$ über dem Fibulaköpfchen, Amplitude 3,2 mV). Im EMG an allen vier Extremitäten Faszikulationen, ansonsten aber keine pathologische Spontanaktivität, kein chronisch-neurogener Umbau. Es erfolgt eine repetitive Stimulation des N. tibialis mit Ableitung (Oberflächenelektroden) am M. abductor hallucis rechts ( $\triangleright$ Abb. 1) [20]. Vor allem nach höherfrequenter Stimulation gelingt der Nachweis von Nachentladungen („afterdischarges“).

In den Laboruntersuchungen zeigte sich bis auf eine leichte Erhöhung der Kreatinkinase (CK) mit 252 U/I (Normwert: < $190 \mathrm{U} / \mathrm{I}$ ) kein wegweisend pathologischer Befund. Antikörper gegen den VGKC-Komplex negativ. Auch eine umfangreiche autoimmunologische (inkl. Acetylcholin-Rezeptor-Antikörper, ANA, cANCA, pANCA, Antiphospholipid-Antikörper) und endokrinologische Diagnostik zeigt keine Auffälligkeiten.

\section{Diskussion}

Das MFKS gehört zu den Hyperexzitabilitätssyndromen des peripheren Nervensystems und zeigt einen benignen Verlauf [8]. Trotzdem können vor allem die Faszikulationen als störend empfunden werden, sodass ein gewisser Leidensdruck entsteht. Therapeutisch stehen v.a. membranstabilisierende Medikamente wie Carbamazepin und Gabapentin zur Verfügung [20, 23]. Die Indikation zu einer Therapie mit Chininsulfat gegen Krampi sollte wegen des schwerwiegenden Nebenwirkungspotenzials zurückhaltend gestellt werden [24].

Differenzialdiagnostisch muss das MFKS als Ausschlussdiagnose gegen zahlreiche andere neurologische Störungen, v.a. Motoneuron-Erkrankungen, abgegrenzt werden. Diagnostisch hilfreich kann eine repetitive Stimulation sein, die insbesondere unter höherfrequenter Reizung Nachentladungen („afterdischarges“) als Ausdruck der Hyperexzitabilität zeigt $[12,20]$.

Der dargestellte Fallbericht illustriert den typischen Verlauf eines MFKS mit benignen Faszikulationen, oft über viele Jahre, ohne dass neurologische Auffälligkeiten auftreten. Vor allem bei Angehörigen des Gesundheitssystems treten dennoch nicht selten Ängste vor einer ALS auf [7], denen man durch die korrekte Diagnosestellung und Aufklärung über die Gutartigkeit des MFKS begegnen kann. 


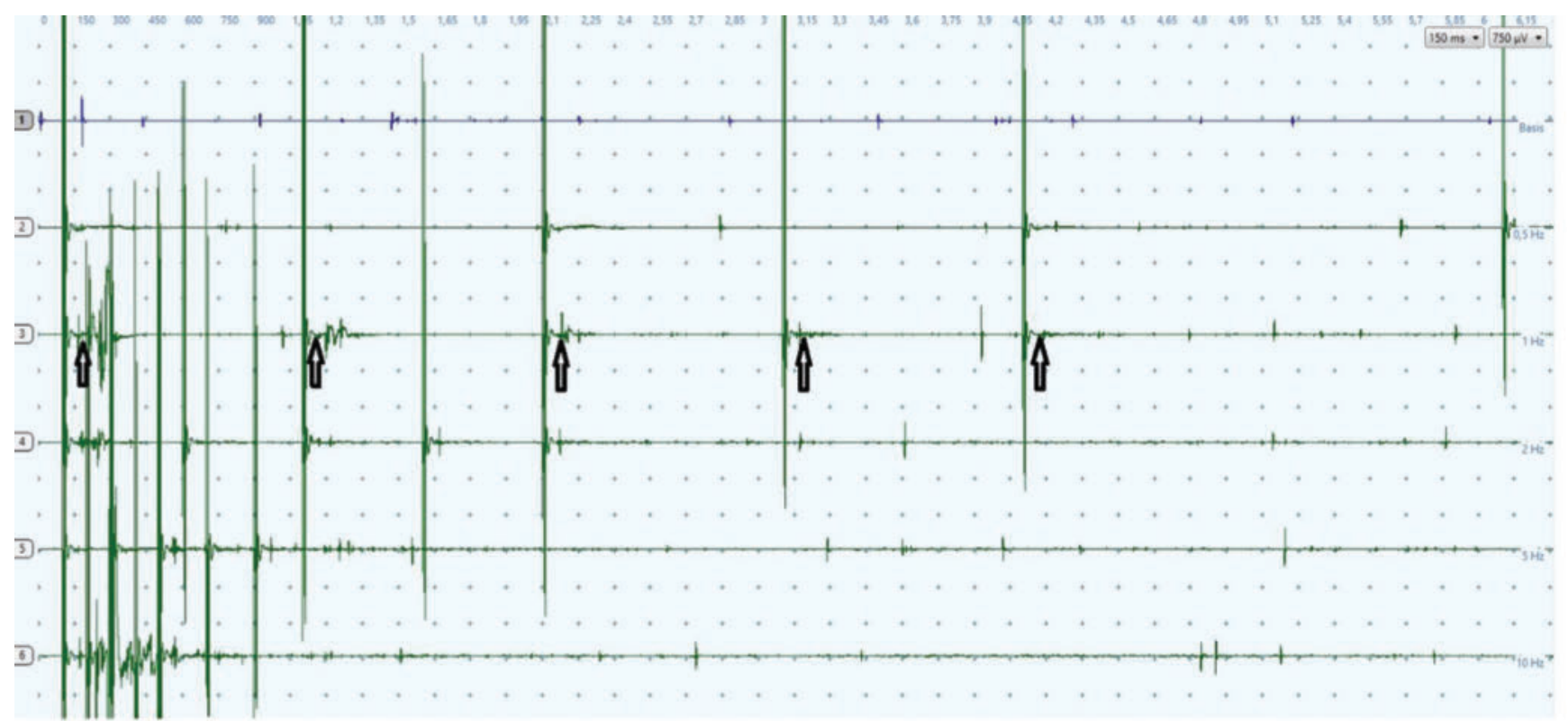

Abb. 1 Repetitive Stimulation des N. tibialis rechts mit Ableitung über dem M. abductor hallucis (Oberflächenelektroden). In Ruhe (Kurve 1) zeigen sich einige Faszikulationen. In der repetitiven Stimulation, z. B. mit $1 \mathrm{~Hz}$ (Kurve 3), treten Nachentladungen („afterdischarges“) auf, die aus didaktischen Gründen mit einem Pfeil gekennzeichnet sind. Unter hochfrequenter Stimulation mit $10 \mathrm{~Hz}$ (Kurve 6) sind diese Nachentladungen ebenfalls gut zu beobachten.

$\begin{array}{ll}\text { ABKÜRZUNGSVERZEICHNIS } \\ \text { CANCA } & \text { cellular anti-neutrophil cytoplasmic antibodies } \\ \text { CASPR2 } & \text { contactin-associated protein-like } 2 \\ \text { CK } & \text { Kreatinkinase } \\ \text { EMG } & \text { Elektromyografie } \\ \text { FP } & \text { Faszikulationspotenziale } \\ \text { MFKS } & \text { Myalgie-Faszikulations-Krampus-Syndrom } \\ \text { MSAP } & \text { Muskel-Summenaktionspotenzial } \\ \text { NMDA } & \text { N-Methyl-D-Aspartat } \\ \text { PANCA } & \text { perinuclear anti-neutrophil cytoplasmic } \\ & \text { antibodies } \\ \text { SNAP } & \text { Sensibles Nervenaktionspotenzial } \\ \text { TRPA1 } & \text { transient receptor potential ankyrin A1 } \\ \text { VGKC } & \text { voltage gated potassium channel }\end{array}$

Interessenkonflikt

Die Autoren geben an, dass kein Interessenkonflikt besteht.

Literatur

[1] Denny-Brown D, Foley JM. Myokymia and the benign fasciculation of muscular cramps. Trans Assoc Am Phys 1948; 61: 88-96

[2] Kottlors M, Glocker FX. Myopathien und neuromuskuläre Erkrankungen. In: Hufschmidt A, Lücking CH, Rauer S, Hrsg. Neurologie compact. 6. Aufl. Stuttgart: Thieme ; 2013: 547-576

[3] Bischoff C, Schulte-Mattler W], Conrad B. Das EMG-Buch. 2. Aufl. Stuttgart: Thieme ; 2005: 209-212

[4] Mills KR. Characteristics of fasciculations in amyotrophic lateral sclerosis and the benign fasciculation syndrome. Brain 2010; 133: 3458-3469
[5] Fermont ], Arts IM, Overeem S et al. Prevalence and distribution of fasciculations in healthy adults: Effect of age, caffeine consumption and exercise. Amyotroph Lateral Scler 2010; 11: 181-186

[6] Mitsikostas DD, Karandreas N, Coutsopetras P et al. Fasciculation potentials in healthy people. Muscle Nerve 1998; 21: 533-535

[7] Simon NG, Kiernan MC. Fasciculation anxiety syndrome in clinicians. J Neurol 2013; 260: 1743-1747

[8] Orsini M, Sztajnbok FR, Oliveira AB et al. Benign fasciculations and corticosteroid use: possible association? An update. Neurol Int 2011; 3: e11

[9] Finsterer J, Aliyev R. Fasciculations in human hereditary disease. Acta Neurol Belg 2015; 115: 91-95

[10] Kiernan MC, Hart IK, Bostock H. Excitability properties of motor axons in patients with spontaneous motor unit activity. J Neurol Neurosurg Psychiatry 2001; 70: 56-64

[11] Nagado T, Arimura K, Sonoda $Y$ et al. Potassium current suppression in patients with peripheral nerve hyperexcitability. Brain 1999; 122: 2057-2066

[12] Liewluck T, Klein C], Jones Jr. LK Cramp-fasciculation syndrome in patients with and without neural autoantibodies. Muscle Nerve 2014; 49: 351-356

[13] Deymeer F, Akca S, Kocaman G et al. fasciculations, autonomic symptoms and limbic encephalitis: a thymoma-associated Morvan's-like syndrome. Eur Neurol 2005; 54: 235-237

[14] Lancaster E, Huijbers MG, Bar V et al. Investigations of caspr2, an autoantigen of encephalitis and neuromyotonia. Ann Neurol 2011; 69: 303-311

[15] Nirenberg M], Chaouni R, Biller TM et al. A novel TRPA1 variant is associated with carbamazepine-responsive cramp-fasciculation syndrome. Clin Genet 2018; 93: 164-168

[16] Fleet WS, Watson RT. From benign fasciculations and cramps to motor neuron disease. Neurology 1986; 36: 997-998

[17] de Carvalho M, Swash M. Cramps, muscle pain, and fasciculations: not always benign? Neurology 2004; 63: 721-723

[18] de Carvalho M, Swash M. Fasciculation-cramp syndrome preceding anterior horn cell disease: an intermediate syndrome? J Neurol Neurosurg Psychiatry 2011; 82: 459-461 
[19] Blexrud MD, Windebank AJ, Daube JR. Long-term follow-up of 121 patients with benign fasciculations. Ann Neurol 1993; 34: 622-625

[20] Tahmoush A], Alonso RJ, Tahmoush GP et al. Cramp-fasciculation syndrome: A treatable hyperexcitable peripheral nerve disorder. Neurology 1991; 41: 1021-1024

[21] Harrison TB, Benatar M. Accuracy of repetitive nerve stimulation for diagnosis of the cramp-fasciculation syndrome. Muscle Nerve 2007; 35: 776-780
[22] Poyraz M, Matur Z, Aysal F et al. Clinical, electrophysiological, and serological evaluation of patients with Cramp-Fasciculation Syndrome. Noro Psikiyatr Ars 2017; 54: 183-186

[23] Forcelini CM, Rotta FT, Posenato $\mathrm{N}$ et al. Benign fasciculations responsive to gabapentin. Arq Neuropsiquiatr 2007; 65: 1015-1017

[24] Arznei-Telegramm. Chinin (Limptar N): Lebensbedrohliche Schadwirkungen. a-t 2010; 41: 99

[25] Ansell J, Kirby S, Benstead T. A case of Isaacs' syndrome with associated central nervous system findings. Muscle Nerve 1997; 20: 1324-1327 